\title{
Micro-valences: perceiving affective valence in everyday objects
}

\author{
Sophie Lebrecht ${ }^{1}$, Moshe Bar ${ }^{2,3}$, Lisa Feldman Barrett ${ }^{2,3,4}$ and Michael J. Tarr ${ }^{5 *}$ \\ 1 Department of Cognitive, Linguistic and Psychological Sciences, Brown University, Providence, RI, USA \\ 2 Department of Psychiatry, Massachusetts General Hospital/Harvard Medical School, Boston, MA, USA \\ ${ }^{3}$ Martinos Center for Biomedical Imaging, Massachusetts General Hospital/Harvard Medical School, Boston, MA, USA \\ ${ }^{4}$ Department of Psychology, Northeastern University, Boston, MA, USA \\ ${ }^{5}$ Department of Psychology, Center for the Neural Basis of Cognition, Carnegie Mellon University, Pittsburgh, PA, USA
}

\section{Edited by:}

Arnon Cahen, Ben Gurion University

of the Negev, Israel

Michela C. Tacca, Heinrich-Heine

University Düsseldorf, Germany

Reviewed by:

Karla K. Evans, Harvard Medical

School, USA

Ian Krajbich, University of Zurich,

Switzerland

\section{${ }^{*}$ Correspondence}

Michael J. Tarr, Center for the Neural Basis of Cognition, Carnegie Mellon

University, 115 Mellon Institute, 4400

Fifth Avenue, Pittsburgh, PA 15213,

USA.

e-mail:michaeltarr@cmu.edu
Perceiving the affective valence of objects influences how we think about and react to the world around us. Conversely, the speed and quality with which we visually recognize objects in a visual scene can vary dramatically depending on that scene's affective content. Although typical visual scenes contain mostly "everyday" objects, the affect perception in visual objects has been studied using somewhat atypical stimuli with strong affective valences (e.g., guns or roses). Here we explore whether affective valence must be strong or overt to exert an effect on our visual perception. We conclude that everyday objects carry subtle affective valences - "micro-valences" - which are intrinsic to their perceptual representation.

Keywords: affective valence, visual object perception, object recognition, micro-valence, object preference

\section{INTRODUCTION}

While grabbing a coffee mug from the cupboard a phone call diverts your attention and, in the split second before answering the call, you select a seemingly random mug from your collection. What factors guide you to choose one mug over the others? Here we answer this question by proposing that the majority of physical objects around us possess a subtle valence - a "micro-valence" which ranges in magnitude but is always present. Although it is certainly the case that others have made similar proposals (Lang et al., 1998; Cunningham et al., 2004; Barrett and Bar, 2009; Colibazzi et al., 2010), the majority of valence studies have focused on objects with strong valence (e.g., guns or roses). More importantly, to the extent that everyday objects automatically evoke some perception of valence, valence can be considered a higher-level object property that connects vision to behavior. In this sense, as with other higherorder properties of objects, we suggest that valence is automatically perceived and incorporated into the mental representations of objects (Figure 1). One implication of this claim is that "standard" models of object perception may need to be rethought (Ungerleider and Mishkin, 1982; Serre et al., 2007). Moreover, by exploring high-level, functional object properties such as valence, we gain a better understanding of how our perceptual system translates visual information into tangible cues for behavior.

Assigning affective values to mental constructs is not a new concept. Social psychologists have been investigating "attitude objects" for decades (Fazio et al., 1986, 1995; Bargh et al., 1992; Greenwald et al., 1998; Giner-Sorolla et al., 1999). Attitude objects can be conceptualized as a person, place, concept, word, or thing that is readily associated with a particular attitude from memory (e.g., associating "fox hunting" with cruelty, or "yoga" with peacefulness). Although related, our conceptualization of micro-valence differs in that we are not focused on individuals' cognitive attitudes to concepts, but rather on the mechanisms underlying how the visual system computes affective valence from perceptual representations. Thus, valence is not a label or judgment applied to the object postrecognition, but rather an integral component of mental object representations. While one might argue that valence is too abstract to be considered an object property, we should note that higher-order properties such as material (Cant and Goodale, 2007; Cant et al., 2008), category (Grill-Spector and Kanwisher, 2005), and function (Beauchamp et al., 2002) are also inferred from multi-modal and non-perceptual sources.

Research investigating the links between affect and perception most commonly relies on object, face, or scene stimuli that generate strong, well-defined valences (Greenwald et al., 1998; Avero and Calvo, 2006; Calvo and Avero, 2008; Rudrauf et al., 2008; Colibazzi et al., 2010; Weierich et al., 2010). In contrast, few studies have examined how more subtle valences are perceived in common objects (e.g., lamps, clocks, or coffee cups; McManus, 1980; Giner-Sorolla et al., 1999; Rentschler et al., 1999; Duckworth et al., 2002; Bar et al., 2006; Bar and Neta, 2007). Of late, studies examining weaker valences in the form of the value placed on everyday objects, such as snack foods (Constantino and Daw, 2010; Krajbich et al., 2010; Litt et al., 2011), have been arisen due to interest in "neuroeconomics." Still, studies relying on extreme responses to highly affective stimuli remain our primary source for understanding the role of affect in perception and cognition. Although critical for outlining the core components of affective processing, 
we suggest that the field should take a more fine-grained look at how subtle affective valences influence our everyday perceptions and interactions with the world.

Affect itself is commonly defined along two continuous dimensions: valence (pleasantness) and arousal (activation; Russell, 1980; Russell and Carroll, 1999; Barrett, 2006). With respect to microvalences we are primarily focused on the single dimension of valence. Although this approach assumes that valence is continuous, we should emphasize that there are still notable differences in the intensity of and the variance in one's responses to strongly and weakly valenced objects, for example, a bloody weapon and a coffee mug. A bloody weapon will prompt a highly intense affective

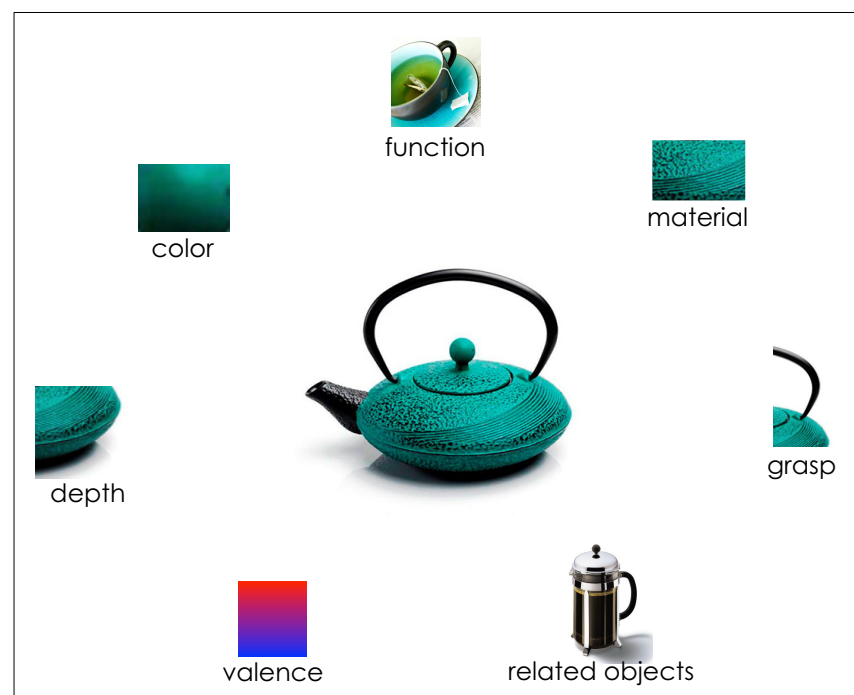

FIGURE 1 | A common, everyday object and variety of high-level object properties that may be components of its mental representation. response in most individuals and the between-subject variance for these strong responses is expected to be relatively low. That is, the vast majority of us will experience the same highly negative feeling when viewing such a negative stimulus. In contrast, a coffee mug will likely generate a weaker or more subtle response, which we refer to as that object's micro-valence. This valence is described as "micro" because the intensity of the response is less than the bloody weapon or other similarly strongly affective objects. However, this weak intensity should not be confused with a weak effect. There are many small, yet robust effects, for example Sternberg's (1966) classic digit memory search exhibited an effect of less than $40 \mathrm{~ms}$ per an item in memory (Sternberg, 1966).

\section{THE PREVALENCE OF VALENCE}

Affective valence is highly prevalent in our visual environment. That is, objects in a scene prompt the perception of valence that varies in strength and intensity along the valence continuum (Figure 2A). As mentioned, while construing valence as a continuous dimension is not a new idea (Russell, 1980; Russell and Carroll, 1999; Colibazzi et al., 2010), many theorists have focused on strongly valenced objects at the extreme ends of the continuum $^{1}$. Here we focus on the subtle differences in microvalence for objects nearer to the center of the valence continuum. We posit that observers perceive a subtle valence for objects even in the region immediately surrounding neutral. That is, everyday objects such as chairs and clocks possess a micro-valence and so are either slightly preferred or anti-preferred. Indeed, when the

\footnotetext{
${ }^{1}$ By way of comparison, when researchers working within the framework of attitude objects refer to a continuum they are typically referring to the continuous relationship between associated strength and automaticity (Fazio et al., 1986). This means that attitude objects vary in the level of associated strength between the attitude and the "object." This continuous relationship is then used to predict the speed that an attitude can be automatically activated from memory.
}

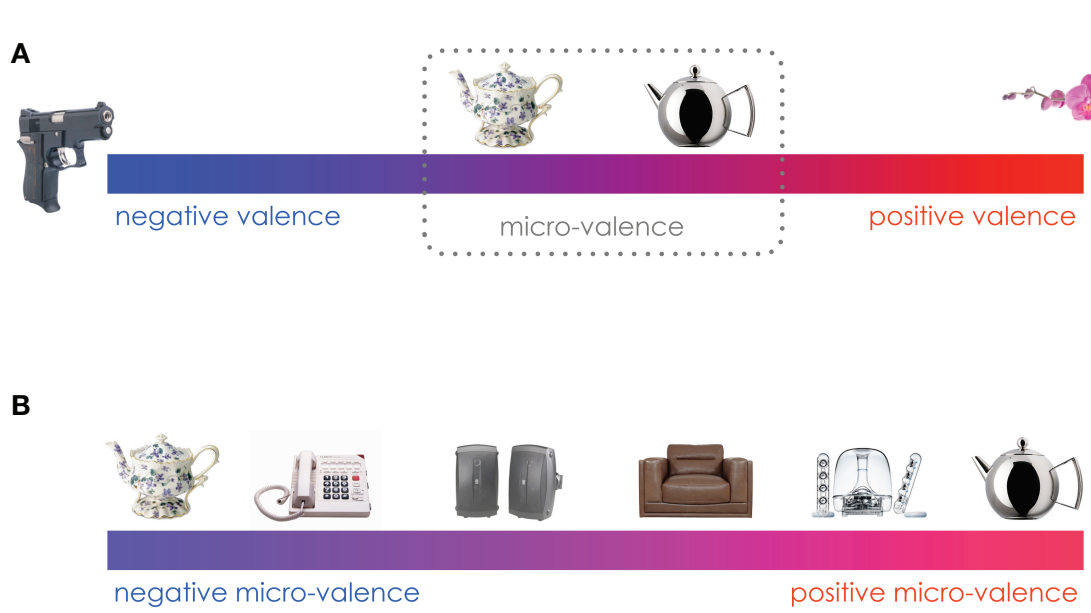

FIGURE 2 |The valence continuum in (A) illustrates the dimension of valence ranging from strongly positive (red) to strongly negative (blue). As indicated by the dashed gray line, objects perceived to have a valence close to the neutral point on the continuum are nonetheless regarded as having a micro-valence. In
(B) the portion of the continuum encompassed by the gray line in (A) has been expanded to represent a finer-grained continuum. The ordering of objects here reflects this expanded continuum, albeit with lesser magnitudes (the ordering of objects in this figure is derived from Lebrecht and Tarr, 2010). 
scale at the center of the continuum is expanded we expect it to be organized much like the larger continuum, the only difference being the overall magnitude of valence is weaker at the center than at the extreme ends (Figure 2B; Lebrecht and Tarr, 2010). Note that this model does not preclude the presence of some objects happening to fall exactly at the center point of the continuum and therefore giving rise to a truly neutral perception of valence.

While micro-valence only accounts for a small absolute region of the valence continuum, it is integral in the automatic perception of objects. As such, "micro" valences are a big issue for understanding visual perception. That is, while it is undoubtedly the case that explicating the processing of strongly affective objects is useful in anchoring how affect and perception might interact, we rarely encounter blocks of gold or blood stained weapons in everyday life. Even if we are to acknowledge that valence functions along a continuum whereby subtle valences are possible, further research is necessary to elucidate the characteristics of the subtle valences that populate the central region of this space.

\section{UNDERSTANDING THE ORIGINS OF MICRO-VALENCE}

Understanding how everyday objects acquire a micro-valence forms a critical aspect of our theory. During perception visual objects evoke a rich set of information (not necessarily affective), including properties that are not an intrinsic feature of the percept or the percept's larger category. For example, seeing an object can automatically activate an entire network of information from memory (Bar, 2007). This network is derived from both contextual experiences (Bar et al., 2008) as well as conceptual or semantic knowledge (Martin et al., 1996; Haxby et al., 2001; Patterson et al., 2007). An operational example of this idea can be seen in the efforts by historians to tell the history of the world in only 100 objects (Schama, 2010): by selecting and describing key objects that elicit a wide range of associations, historians were able to evoke critical periods in history. For example, one particular object, the Roman Samian Bowl (Balmuildy Fort, Antonine Wall - second century AD), was bright red, engraved with human, animal, and floral figures. The bowl, made in a workshop in France, was thought to have been used at banquets for Roman officers and other high status individuals. The information attributed to this object told a story of craftsmanship and Roman rule in France in 200 AD. Historians regard objects as loci of archival information acting as an access point for vast quantities of knowledge and experience. In much the same way, we perceive a richer network of information than that immediately available in the percept. Our perception of any common object automatically accesses a large network of information in our memories - valence is only one component of a wide array of information, including semantic and conceptual information that arises during object perception.

It is these associations, spontaneously reactivated during perception, that help form what becomes an object's micro-valence. For example, the principle that the contextual experience with an object informs the micro-valence is evident in the micro-valence of telephones: phones that resembled those used in the bedroom were rated as more positive in valence than phones were used in the office (Lebrecht and Tarr, 2010). Moreover, these and other micro-valence ratings obtained in our studies have been found to be consistent both within and across observers, thereby indicating that micro-valence is more than random variation around a neutral mean (Lebrecht and Tarr, 2010).

What makes the derivation of micro-valence much more complex is that we have typically seen an object in many different contexts and with a variety of individuals, all of which will contribute to the affective memories or associations that become automatically reactivated during visual object perception. Microvalence is the aggregate of this information plus, as discussed below, valence-relevant perceptual object properties. This cumulative valence - the contextual component being accrued over our personal experiences - determines, in part, whether a given object is ultimately preferred (having positive micro-valence) or anti-preferred (having negative micro-valence).

Supporting this framework, there is strong evidence suggesting that individuals rapidly attribute valence information to objects, which then influences subsequent perceptions (Zajonc and Markus, 1982; Murphy and Zajonc, 1993; Duckworth et al., 2002; Ghuman and Bar, 2006; Bliss-Moreau et al., 2008). Such findings indicate that individuals are able to associate affective information with objects, which can then bias perception at a later point in time.

\section{THE PERCEPTUAL COMPONENTS OF MICRO-VALENCE}

Although micro-valences appear to be "high-level," there is evidence suggesting that low-level visual properties contribute to an object's perceived valence. That is, visual features such as shape, curvature, color, material, and symmetry may add to an object being perceived as positive or negative independent of affective associations.

Experimentally, this question has been addressed by examining novel objects for which there are few pre-existing associations. For example, when making rapid "gut reaction" judgments participants consistently prefer curved over sharp or jagged objects for both familiar and novel objects (Bar et al., 2006). Other studies have observed that participants can make valence judgments on simple shapes (McManus, 1980; Rentschler et al., 1999; Bar et al., 2006). At the same time, several studies report more reliable ratings for real-world images as compared to abstract shapes (Vessel and Rubin, 2010), indicating that experience-based associations are dominant in forming valences.

More plausibly micro-valences arise from an integration of visual properties and learned associations. Moreover, these two attributes may potentially interact in that it may be easier to form positive associations with objects already possessing "positive" perceptual features. Consider that an observer might more readily generate positive associations with a shiny, curved, symmetrical teapot, whereas the same observer might more readily generate negative associations with a dull, angular, asymmetric teapot. Conversely, there is some evidence that this interaction between perceptual features and associations may also function in reverse: data to suggest that color preference might sometimes arise from the degree to which an individual prefers an object with a particular color, so that participants would be more likely to prefer green to yellow if they prefer apples to bananas (Palmer and Schloss, 2010). 


\section{BEYOND VALENCE}

It is our view that valence should be construed as a property of object representations. As such, valence contributes to and is correlated with, but is not equivalent to, either aesthetics or preference. With respect to the former, object valence is closely related to visual aesthetics. Whereas we are focused on the singular dimension of valence in perception, aesthetics can be broadly defined as a discourse of how works of art are judged to be beautiful across different eras (Baumgarten, 1750; Ramachandran and Hirstein, 1999; Kirk et al., 2009). Thus, how our visual system evaluates objects or images as positive or negative may contribute to aesthetic experience, but cannot account for its entirety. Valence therefore can be considered but one aspect of aesthetic experience. More closely related to micro-valence is the emerging field of everyday aesthetics (Mandoki, 2007; Saito, 2007), which addresses the ramifications of making aesthetic judgments on everyday environments, for example, the ecological impact of maintaining lush green lawns because they are aesthetically pleasing.

As with aesthetics, it is our perspective that valence is but one component of preference, and not actually preference per se. Preference is defined at the level of behavior (Lichtenstein and Slovic, 2006). For example, if you select a cappuccino over an espresso you are held to have a preference for cappuccinos. By the time you reach this level of behavior an array of mental processes have been deployed: the object has been perceived, the relative valences of objects in the scene have been computed, these valences have been compared one's current goals and motivations in the specific context (Constantino and Daw, 2010; Krajbich et al., 2010; Litt et al., 2011), and these factors are combined to produce the current "value" of the objects (Rangel et al., 2008; Grabenhorst and Rolls, 2011). Only then can an individual make a decision and in doing so exhibit preference. As such, perceiving valence in objects is only one component of preference formation and, although valence and preference are often highly correlated, they are not the same mental construct. For example, a person may perceive one cell phone to possess a more positive micro-valence than the other, yet may ultimately buy the cell phone with the negative micro-valence because it has better features. Within our

\section{REFERENCES}

Avero, P., and Calvo, M. G. (2006). Affective priming with pictures of emotional scenes: the role of perceptual similarity and category relatedness. Span. J. Psychol. 9, 10-18.

Bar, M. (2007). The proactive brain: using analogies and associations to generate predictions. Trends Cogn. Sci. (Regul. Ed.) 11, 280-289.

Bar, M., Aminoff, E., and Schacter, D. L. (2008). Scenes unseen: the parahippocampal cortex intrinsically subserves contextual associations, not scenes or places per se. J. Neurosci. 28, 8539-8544.

Bar, M., and Neta, M. (2007). Visual elements of subjective preference modulate amygdala activation. Neuropsychologia 45, 2191-200.

framework, valence is a positive or negative property of object representations that is computed automatically by our visual systems, whereas preference is an active choice behavior.

\section{DISCUSSION}

The functional significance of objects with strong valence is highly intuitive; we dislike objects that indicate danger, threat, or disgust, such as angry tigers or moldy food, and we like objects that indicate sustenance or pleasure, such as appetizing food or attractive mates. But why have we also come to automatically evaluate the valence of everyday objects that appear neither threatening nor life promoting?

We contend that micro-valences function to optimize our ability to either select or orientate toward objects with a positive micro-valence and away from those with a negative micro-valence. Throughout the day we make multiple unconscious decisions: what mug to use for our morning coffee, what pen to sign with, and what bottle of water to purchase. We suggest that these decisions are facilitated by micro-valences computed during perception, which we can use to reduce uncertainty and/or to orientate toward some objects and away from others.

Not surprisingly, these issues have not been lost on the product design and marketing communities. For example, Donald Norman, an expert in the psychology of product design, has argued that affective properties, or in our terms, micro-valence, enhance the usability of particular objects (Norman, 2003).

In summary, we contend that our perception of the world is always colored by our experiences and predispositions. We are social creatures that, through a variety of contextual experiences, create a visual world animated with affect. As observers we must decode the multitude of perceptual, affective, and semantic information presented to our senses. To solve the affect part of this equation we evaluate the valence of all visual objects across the scene. Much in the same way that we automatically perceive the shape, size, or color of objects, we cannot help but perceive the valence in objects. In this sense, valence is not a label applied after the fact to perceptual entities, but rather is an intrinsic element of visual perception with the same mental status as other object properties.

streams for manipulable objects and human movements. Neuron 34, 149-159.

Bliss-Moreau, E., Barrett, L. F., and Wright, C. I. (2008). Individual differences in learning the affective value of others under minimal conditions. Emotion 8, 479-493.

Calvo, M. G., and Avero, P. (2008). Affective priming of emotional pictures in parafoveal vision: left visual field advantage. Cogn. Affect. Behav. Neurosci. 8, 41.

Cant, J. S., and Goodale, M. A. (2007). Attention to form or surface properties modulates different regions of human occipitotemporal cortex. Cereb. Cortex 17, 713-731.

Cant, J. S., Large, M. E., McCall, L., and Goodale, M. A. (2008). Independent processing of form, colour, and texture in object perception. Perception 37, 57-78.

Colibazzi, T., Posner, J., Wang, Z., Gorman, D., Gerber, A., Yu, S., and Peterson, B. S. (2010). Neural systems subserving valence and arousal during the experience of induced emotions. Emotion 10, 377-389.

Constantino, S. M., and Daw, N. D. (2010). A closer look at choice. Nat. Neurosci. 13, 1153-1154.

Cunningham, W. A., Raye, C. L., and Johnson, M. K. (2004). Implicit and explicit evaluation: fMRI correlates of valence, emotional intensity, and control in the processing of attitudes. J. Cogn. Neurosci. 16, 1717-1729.

Duckworth, K. L., Bargh, J. A., Garcia, M., and Chaiken, S. (2002). 
The automatic evaluation of novel stimuli. Psychol. Sci. 13, 513-519.

Fazio, R. H., Jackson, J. R., Dunton, B. C., and Williams, C. J. (1995). Variability in automatic activation as an unobtrusive measure of racial attitudes: a bona fide pipeline? J. Pers. Soc. Psychol. 69, 1013-1027.

Fazio, R. H., Sanbonmatsu, D. M., Powell, M. C., and Kardes, F. R. (1986). On the automatic activation of attitudes. J. Pers. Soc. Psychol. 50, 229-238.

Ghuman, A. S., and Bar, M. (2006). The influence of nonremembered affective associations on preference. Emotion 6, 215-223.

Giner-Sorolla, R., García, M. T., and Bargh, J. A. (1999). The automatic evaluation of pictures. Soc. $\operatorname{Cog} n .17$, 76-96.

Grabenhorst, F., and Rolls, E. T. (2011). Value, pleasure and choice in the ventral prefrontal cortex. Trends Cogn. Sci. (Regul. Ed.) 15, 56-67.

Greenwald, A. G., McGhee, D. E., and Schwartz, J. L. (1998). Measuring individual differences in implicit cognition: the implicit association test. J. Pers. Soc. Psychol. 74, 1464-1480.

Grill-Spector, K., and Kanwisher, N. (2005). Visual recognition: as soon as you know it is there, you know what it is. Psychol. Sci. 16, 152-160.

Haxby, J. V., Gobbini, M. I., Furey, M. L., Ishai, A., Schouten, J. L., and Pietrini, P. (2001). Distributed and overlapping representations of faces and objects in ventral temporal cortex. Science 293, 2425-2430.

Kirk, U., Skov, M., Hulme, O., Christensen, M. S., and Zeki, S. (2009). Modulation of aesthetic value by semantic context: an fMRI study. Neuroimage 44, 1125-1132.
Krajbich, I., Armel, C., and Rangel, A. (2010). Visual fixations and the computation and comparison of value in simple choice. Nat. Neurosci. 13, 1292-1298.

Lang, P. J., Bradley, M. M., Fitzsimmons, J. R., Cuthbert, B. N., Scott, J. D., Moulder, B., and Nangia, V. (1998). Emotional arousal and activation of the visual cortex: an fMRI analysis. Psychophysiology 35, 199-210.

Lebrecht, S., and Tarr, M. (2010). Defining an object's micro-valence through implicit measures. J. Vis. 10, 966.

Lichtenstein, S., and Slovic, P. (2006) The Construction of Preference. Cambridge, NY: Cambridge University Press.

Litt, A., Plassmann, H., Shiv, B., and Rangel, A. (2011). Dissociating valuation and saliency signals during decision-making. Cereb. Cortex 21, 95-102.

Mandoki, K. (2007). Everyday Aesthetics: Prosaics, the Play of Culture and Social Identities. Burlington, VT: Ashgate.

Martin, A., Wiggs, C. L., Ungerleider, L. G., and Haxby, J. V. (1996). Neural correlates of category-specific knowledge. Nature 379, 649-652.

McManus, I. C. (1980). The aesthetics of simple figures. Br. J. Psychol. 71(Pt 4), 505-524

Murphy, S. T., and Zajonc, R. B. (1993). Affect, cognition, and awareness: affective priming with optimal and suboptimal stimulus exposures. J. Pers. Soc. Psychol. 64, 723-739.

Norman, D. A. (2003). Emotional Design: Why We Love (or Hate) Everyday Things, 1st Edn. New York: Basic Books.

Palmer, S. E., and Schloss, K. B. (2010). An ecological valence theory of human color preference. Proc. Natl. Acad. Sci. U.S.A. 107, 8877-8882.
Patterson, K., Nestor, P. J., and Rogers, T. T. (2007). Where do you know what you know? The representation of semantic knowledge in the human brain. Nat. Rev. Neurosci. 8 , 976-987.

Ramachandran, V. S., and Hirstein, W. (1999). The science of art: a neurological theory of aesthetic experience. J. Conscious. Stud. 6, 15-51.

Rangel, A., Camerer, C., and Montague, P. R. (2008). A framework for studying the neurobiology of value-based decision making. Nat. Rev. Neurosci. 9, 545-556.

Rentschler, I., Jüttner, M., Unzicker, A., and Landis, T. (1999). Innate and learned components of human visual preference. Curr. Biol. 9, 665-671.

Rudrauf, D., David, O., Lachaux, J. P., Kovach, C. K., Martinerie, J., Renault, B., and Damasio, A. (2008). Rapid interactions between the ventral visual stream and emotionrelated structures rely on a twopathway architecture. J. Neurosci. 28, 2793-2803.

Russell, J. A. (1980). A circumplex model of affect. J. Pers. Soc. Psychol. 39, 1161-1178.

Russell, J. A., and Carroll, J. M. (1999). On the bipolarity of positive and negative affect. Psychol. Bull. 125 3-30.

Saito, Y. (2007). Everyday Aesthetics. Oxford, NY: Oxford University Press.

Schama, S. (2010). What objects say about our times. Financial Times.

Serre, T., Wolf, L., Bileschi, S., Riesenhuber, M., and Poggio, T. (2007). Robust object recognition with cortexlike mechanisms. IEEE Trans. Pattern Anal. Mach. Intell. 29, 411-426.

Sternberg, S. (1966). High-speed scanning in human memory. Science 153 , 652.
Ungerleider, L. G., and Mishkin, M. (1982). "Two cortical visual systems," in Analysis of Visual Behavior, eds D. J. Ingle, M. A. Goodale, and R. J. W. Mansfield (Cambridge, MA: The MIT Press), 549-586.

Vessel, E. A., and Rubin, N. (2010). Beauty and the beholder: highly individual taste for abstract, but not real-world images. J. Vis. 10, 18.118.14.

Weierich, M. R., Wright, C. I., Negreira, A., Dickerson, B. C., and Barrett, L. F. (2010). Novelty as a dimension in the affective brain. Neuroimage 49, 2871-2878.

Zajonc, R. B., and Markus, H. (1982). Affective and cognitive factors in preferences. J. Consum. Res. 9, 123-131.

Conflict of Interest Statement: The authors declare that the research was conducted in the absence of any commercial or financial relationships that could be construed as a potential conflict of interest.

Received:07 November 2011; accepted: 24 March 2012; published online: 17 April 2012.

Citation: Lebrecht S, Bar M, Barrett LF and Tarr MJ (2012) Micro-valences: perceiving affective valence in everyday objects. Front. Psychology 3:107. doi: 10.3389/fpsyg.2012.00107

This article was submitted to Frontiers in Perception Science, a specialty of Frontiers in Psychology.

Copyright (c) 2012 Lebrecht, Bar, Barrett and Tarr. This is an open-access article distributed under the terms of the Creative Commons Attribution Non Commercial License, which permits noncommercial use, distribution, and reproduction in other forums, provided the original authors and source are credited. 